\title{
La metafora "inaudita": Orignarietà e paradossia della metafora
}

(La "voce"-phonè-è la metafora di un suono-psophos-inaudito?)

\section{1.}

Maxim Gorki in un suo scritto autobiografico, "Le mie università" ricorda la domanda postagli da un contadino: "Spiegami, come è possibile? Lo sguardo umano percepisce segni che si compongono in parole ed io riconosco in quei tratti le nostre parole viventi! Come accade? Nessuno me le sussurra. Se fossero immagini capirei. Ma qui si vedono per cosí dire, i pensieri stampati! Come è possibile? (M. Gorki, Autobiographische Romane, München 1972, 688).

Il medesimo problema lo poniamo a proposito della lingua: essa consiste in voci (phonai) che a loro volta sono costituite da suoni (psophoi), cioè-per rimanere al testo di Gorki-da quest'ultimi sorgono "le nostre parole viventi." Come mai ne riconosciamo il significato? Ci viene questo "significato" forse sussurrato da qualcuno? Palesemente da nessuno. Allora come avviene la metafora dei suoni, in quanto a questi-come dicevamo-vengono "trasferiti" dei significati, le voci?

Nel tentativo di rispondere a questa domanda seguo un metodo opposto a quello della retorica tradizionale: introduttivamente premetterò le conclusioni alle quali sono giunto per esporre la rispettiva legittimazione argomentativa.

Ricordiamo le prime righe del Peri hermenias, Dell'interpretazione di Aristotele: "La voce (phonè) è simbolo delle passioni dell'anima" (Esti mèn oun ta en te phonè tor ev te pychè pathematon symbola Aristotele, Peri hermenias 16 a 4)

Punto di partenza della metafisica tradizionale dell'occidente è il problema degli enti, del loro significato: tale tradizione si sforza, tramite il processo razionale, di definire l'Essere degli enti. Tale 
metodo appare perfettamente legittimo in quanto gli enti-come questo termine esprime-partecipano, sono cioè "participi” dell'Essere. D'altro canto il chiarimento dell'Essere degli enti può solo avvenire a mezzo di un processo logico, in quanto questo, rifacendosi a valide ragioni, pretende di raggiungere l'oggcttività.

Questo processo logico è essenzialmente astratto-cioè prescinde dal "qui" e dall" "ora" - considerati come elementi relativi-e giunge cosí ad un universale sempre e dovunque valido.

Il trasferire al suono un significato per mezzo di un processo razionale è il metodo di Platone-nella sua interpretazione di Socratemetodo da lui ritenuto necessario per rifiutare le tesi relativistiche, soggettivistiche della sofistica.

Oggi ci chiediamo: questo schema tradizionale del filosofare, che è stato determinante per il pensiero occidentale, è valido?

Il processo logico astrae programmaticamente-con la definizione logica degli enti-dall'apparire "qui" e dall" "ora" degli enti, mentre di fatto noi li "patiamo" nell'ambito di concrete "situazioni." Astrarre da queste-per pretendere in questo modo di giungere alla comprensione dell'Essere degli enti-implica la necessità di un pensare e parlare completamente astratto cioè appunto quello della logica e metafisica tradizionale.

In che ambito si manifestano originariamente gli enti? In quello dei sensi, entro i limiti di piacere e dolore, sensazioni originarie che a loro volta sono la fonte delle passioni e cioè paura di non essere capace di identificare il significato dei fenomeni ("phainomena" da "phainesthai," apparire) speranza di riuscirvi; invidia di fronte a coloro che-a differenza di noi-riescono in tale compito; benevolenza e desiderio di partecipare alla loro opera e realizzare con loro una comunanza di amicizia ed amore.

Teniamo presente che ciò che si manifesta (phainomenon) appare in funzione di "organi," cioè "strumenti": i sensi (visivo, uditivo, olfattivo). Quindi il problema del significato del "sentito" può essere solo risolto dalla conoscenza del "codice" che "schiude" il senso di ciò che appare, codice del quale appunto i sensi sono "strumenti."

Parlo di "codice," un terminc cquivoco oggi e non vorrei essere frainteso: la semiotica usa tale termine in un senso soggettivistico, cioè come "chiave" scelta da un individuo per "decifrare" c quindi "comprendere" i segni della realtà; tale "scelta" viene intesa come 
un atto libero, con il quale si "schiude," si "legge" il significato degli enti.

Il "codice sensitivo" del quale io parlo invece non è soggettivo, non viene scelto ad arbitrio ma viene "patito," sofferto dai sensi, in quanto ciò che essi ci manifestano appare nell'ambito del piacere $\mathrm{e}$ del dolore entro i limiti dei quali-come segni indicativi-si impone originariamente il significato dei "phainomena," delle apparenze sensibili. Noi non abbiamo quindi qui la dualità di codice e realtà da decodificare ma solo l'apparire immediato-nella passione che soffriamo-del sensibile.

In quanto ciò che appare si manifesta entro i limiti di piacere (hedonè) e dolore (lype), i rispettivi fenomeni hanno sempre un carattere passionale, perché il "codice" sensibile, illustrativo, indicativo preme, urge, viene sofferto.

$\mathrm{Nel}$ De anima Aristotele sottolinea che gli elementi del linguaggio (logos), cioè le voci sono suoni semantici (phonai semantikoi): "semantikos gar de tis psophos estin he phonè" (Aristotles, De anima II, 420 b 29). Dobbiamo dunque concludere che le voci hanno un carattere passionale in quanto appaiono entro i limiti di piacere dolore, come segnali indicativi del corrispondere o non corrispondere all'urgenza di cui i sensi sono organi, instrumenti? Ma segni indicativi (a mezzo di organi) di che cosa?

Aristotele distingue fondamentalmente-e vedremo che in tale modo predetermina il destino della metafisica tradizionale-il suono (psophos) dalla voce (phonè) per poi-come abbiamo testé vistodefinire la voce come un suono indicativo (psophos semantikos). Da ciò dovremmo dedurre che la voce costituisce qualcosa di completamente "nuovo" in confronto al suono, non solo, ma che la voce è una "metafora," cioè nasce dal "trasferire" (metapherein) un significato, un segno indicativo (sema) al suono (psophos).

Avremmo dunque con Aristotele il rifiuto della nostra precedente tesi dell'indeducibilità di un originario, patito "orizzonte" misterioso, abissale che si schiude in funzione ad un "codice" sofferto per contrapporvi-da parte di Aristotele — solo la dualità di un codice - che dobbiamo scegliere - c degli enti che vanno interpretati.

Una siffatta concezione tradizionale del "codice" presuppone non solo la dualità di soggetto ed oggetto ma anche l'affermazione sia di un processo razionale causale (causa ed effetto che rivela il codi- 
ce) sia di un processo temporale (prima e poi) in funzione al quale sorge il nostro mondo. Preminenza dunque-in questo caso- del problema del "perché"—e quindi della "spiegazione," cioè del problema della "verità" degli enti-e non del problema di un originario "svelarsi" indicativo dell'Essere degli enti.

Ma quale c̀ l'argomento al quale Aristotele ricorre per dimostrare tale tesi? Essa ha-come subito vedremo-un carattere "meccanico" cioè avviene in funzione di un processo causale riferendosi al susseguirsi di causa ed effetto.

Ma otteniamo in funzione di questo processo la spiegazione della voce, del suono semantico, indicativo?

Ogni spiegazione causale — come abbiamo già messo in rilievo"astrae" dal dato di fatto fondamentale che il suono (psophos) ci si manifesta esclusivamente tramite un organo, cioè uno strumento di un urgere, di un appello che nel suo carattere misterioso vuole essere cor-risposto.

Ripeto: La spiegazione razionale, meccanica non considera, cioè astrae dal fatto che il suono si manifesta in funzione di uno "strumento"-l'organo sensibile-che rinvia a ciò di cui è strumento ed in vista del quale solo se ne può comprendere il significato. Un suono che non appare in funzione dell'organo uditivo non esiste, è una pura astrazione "meccanica," causale, "tecnica."

Appunto perché ciò che si manifesta per mezzo dei sensi, cioè di organi (udito, vista offatto ecc.) appare entro i limiti di piacere e dolore, ottiene sempre-in vista di questi limiti-il suo originario significato passionale, indicativo. Dobbiamo dunque dedurre che non esistono suoni, sapori, odori, colori, senza significato ma sempre e solo "fenomeni" significativi, una tesi che di primo acchito ci sembra assurda.

Il mondo che si sventaglia per mezzo dei sensi è il nostro mondo originario, sono i sensi che aprono il sipario del teatro sul cui palcoscenico appariamo come attori ed al tempo stesso come spettatori.

Non vi sono suoni, luci, sapori senza significati, senza passione e proprio per questa ragione l'originarietà del nostro mondo non può venire ricercata in una spiegazione causale che di fatto si sforza programmaticamente di astrarre dai sensi.

La voce semantica, indicativa-mediante il piacere ed il dolore radica nella profondità di una realtà abissale, originaria: indeducible 
palesarsi, istantaneo, senza un "prima" e "poi," senza distinzione di causa ed effetto, senza "perché." È questo il profondo significato della disperata difesa della voluttà di Lorenzo Valla (De voluptate) cioè della sua difesa del piacere?

Di qui il sorgere della domanda: le precedenti considerazioni legittimano la ulteriore tesi del carattere metaforico del linguaggio (logos) in quanto consta di suoni significativi? Ma allora che senso fa qui parlare di metafora?

Fin dall'inizio, ciò che appare per mezzo dei sensi, si rivela espressione di un continuo "patire," un appello che si identifica e non si identifica con il singolo manifestantesi: ci troviamo di fronte ad un evento originario: l'accadere del mondo del'ordine dell "Kosmos" sensibile.

Se siamo necessitati a riconoscere questo dato di fatto originario, questa presenzialità, questo avvenimento istantaneo, "in-stans," come hic stare, il filosofare può oggi avere solo senso se non parte piú dalla definizione astratta, razionale degli enti bensí dal problema del carattere passionale dei fenomeni sensitivi. Con decisione dobbiamo ammettere la tesi che non esistono per noi fenomeni-suoni, visioni, sapori-astratti dal loro significato passionale ma solo quali appaiono ed urgono come espressione di un mistero che si serve dei sensi per rivelarsi.

Dunque preminenza della passione rispetto alla ragione: in tal modo non si nega affatto la funzione della ragione, sebbene questo problema non possa essere trattato nell'ambito di questo lavoro.

Se la "phonai"- $\mathrm{i}$ suoi indicativi del linguaggio-hanno sempre un carattere originario significante anche l'ulteriore problema del linguaggio (logos) può e deve venire trattato nell'ambito della nostra precedente problematica. Bisognerà rifarsi alla tesi di G.B. Vico dell'orginario carattere metaforico e passionale della lingua.

Accingiamoci alla dimostrazione della precedente tesi. Presupposto delle parole, del linguaggio (logos) sono le voci (phonai). Le nostre seguenti riflessioni vogliono solo essere un tentativo di identificare l'ambito originario del linguaggio ossia avere solo carattere di "prolegomena" al problema del linguaggio.

Aristotele, come abbiamo già riferito, distingue "suono" (psophos) 
da "voce" (phonè) definendo quest'ultima come un suono indicativo (psophos semantikos): "semantikos gàr tis psophos estin he phonè." Aristotele, De anima II 420 b 29).

Da qui nasce il problema: possiamo affermare che il suono sia trasformato in voce (phonè) in quanto gli viene "traslato" un segno indicativo (sema)? In tale caso al sorgere di una voce (phonè) contribuirebbero due elementi: il suono (psophos) e il trasferirgli un significato, una indicazione. $\grave{E}$ in funzione di questi due elementi che sorgerebbe il "nuovo" mondo, il nuovo "kosmos," il "nuovo" ordine, quello del linguaggio.

Prima di negare la legittimità di questo dualismo teniamo presente la concezione tradizionale della metafora.

Anzitutto non va dimenticato che il "traslare" (metapherein) non ha originariamente un sigificato "linguistico" e tanto meno "letterario": il termine "metapherein" indica il "tra-sferire" un oggetto da un luogo ad un altro luogo-dualità-il che presuppone un "passaggio," un "transito," un "ponte" (Erodoto I, 64: Tucidide I, 134, 4), che l'uomo deve "progettare," cioè "gettare" da un luogo "ad un 'altro' luogo," da un "qui" ad un "la," il che a sua volta implica un "prima ed un poi," di una tale attuazione.

Aristotele nella Retorica e nella Poetica definisce la metafora come il traslare del significato di una termine (aquila) da un ambito in un altro ambito (potere). Secondo Quintiliano la metafora è il risultato di una mutazione ("cum virtute mutatio." Quintiliano, Institutiones oratoriae VIII, 6, 1).

Questo concetto della metafora è uno scandalo per la logica in quanto questa pretende di identificare razionalmente i fenomeni: la trasposizione di significati implica una rinuncia alla stressa razionalità.

Tradizionalmente la metafora viene quindi situata nell'ambito di un arte-poesia, retorica- c come tale esclusa dalla filosofia.

Hegel scrive che "l'idea" deve essere libera da qualsiasi sensibilità e rimprovera ad esempio all'umanesimo di ricorrere ad immaginicome la metafora-perché questa è solo qualcosa di "naturale" e come tale non raggiunge l'altezza dal concetto (Hegel, Vorlesungen über die Geschicte der Philosophie, ed. Glockner XVII, Stuttgart 1928, 121, 149).

Di consequenza la metafora risulta-secondo la filosofia tradizio- 
nale-un elemento "di-straente," che non ha nulla a che fare con la realtà, espressione di una attività fantastica, che può tutt'alpiúcome nel pensiero medioevale-essere usata come "integumentum," come "corteccia" della verità razionale per farla accettare a chi è incapace di "pensare rigorosamente." "Fabulas poetac a fando nominaverunt quia non sunt res factac, sed tantum fictac" (Adelardo da Bath, De eodem et diverso IV, 1, 25 in: Beiträge zur Geschichte der Philosophie des Mittelalters IV, 1, S. 27).

Hrabanus Maurus scrive: "Il compito del pocta consiste nel traslare reali avvenimenti in altre forme a mezzo di immagini appropriate." ("Officium poetac in eo est, ut ca, quae vero gesta sunt, in alias species obliquis figurationibus cum decore aliquo conversa transducant." Hrabanus Maurus. De universo XV, II, P. L. III, 419 c).

Metafora e poesia dunque esclusivamente come istrumenti della fantasia che non ha nulla a che fare con la realtà.

È legittima questa concezione della metafora con la quale quest'ultima viene relegata nell'ambito del puramente "letterario" o della "retorica" ed esclusa da ogni considerazione di un pensare "scrio," speculativo? È quindi la metafora semplicemente un "gioco" al quale non può venire riconosciuta alcuna funzione filosofica?

L'ontologia - come scienza razionale degli enti, degli "onta"costituisce per il pensiero tradizionale il presupposto per la scienza dell'Essere, per la Metafisica: con la prima si pretende di raggiungere il "codice" dell'Essere degli essenti. Di qui la tesi dell'identità del problema degli enti con quello dell'Essere primo, originario Ente, cioè con la metafisica.

Anche la teoria della conoscenza della filosofia moderna-che prende avvio dalla cosidetta "rivoluzione copernicana" di Kant-nel suo filosofare parte dagli essenti chiedendosi secondo quali forme della conoscenza essi si rivelano: dualità di oggetto e soggetto, di noumeno e di forma conoscitiva, presupposti di ogni teoria della conoscenza, di ogni gnoseologia, con la conclusione dall'impossibilità di conoscerc il noumeno. Anche nella critica di $\mathrm{Hegel}$ a Kant viene confermata la preminenza del carattere razionale del reale ragione per la quale il profondo significato del reale può e deve venire dedotto a priori con un processo dialettico logico.

Anche qui rifiuto della concezione tradizionale della metafora, e con ciò della poesia perché con I"“Ideale"-secondo la formulazione 
di Hegel-non si raggiunge ancora l'ambito dell'Idea e rifiuto della retorica come un linguaggio essenzialmente relativo perché legato al "qui" ed all'"ora."

Ma veniamo al problema fondamentale che ci interessa: è dunque valida la concezione tradizionale dualistica di suono (psophos) e voce (suono semantico indicativo, psophos semanticos cioè phonè)? Abbiamo un ente sonoro precedente ad ogni significato? Solo rispondendo a questa domanda possiamo accertare se la phonè consiste nel "trasferire," nel "traslare" un significato, un segno indicativo (sema) su un suono oppure, e in che senso la metafora debba essere concepito differentemente come un fenomeno originario ed indeducibile dal suono, assumendo cosí un significato ed una funzione ben piú profonda di quella tradizionale.

Ancora una volta mi riferisco ad Aristotele: egli spiega il suono (psophos) come la conseguenza di un processo meccanico e cioè di una percussione (causa) su un ente, percussione dalla quale nasce una vibrazione (effetto) che per mezzo di un medio-acqua, ariagiunge all'organo uditivo.

Spiegazione causale, razionale. Egli insiste su questo punto tanto da distingere enti sonori e non sonori. "La lana percossa non dà suoni bensí solo i minerali” (Aristotele, De anima II, 419 b13). Si tratta dunque di un processo che gli enti soffrono, spicgazione essenzialmente "meccanica."

A questo riguardo va tenuto presente un sorpredente passo aristotelico al quale raramente si ricorre nell'ambito di questa problematica. "Se è necessario realizzare qualcosa contro la natura (ti para physin praxai) allora sorge una difficoltà (aporia) ed è necessario ricorrere ad una arte (deinai technes): chiamiamo una parte dell'arte (techne) che ci permette di superare una difficoltà (aporia) mechanè" (Aristotele, Mekanica 847 a 13).

Risolviamo il problema della phonè ricorrendo al fenomeno del suono (phonè), alla spiegazione "tecnica" meccanica"? La domanda si impone dato che lo stesso Aristotele afferma che quando realizziamo qualcosa "contro natura"- - cioc̀ contro ciò che nasce, fiorisce e muore-ricorriamo alla spiegazione "meccanica"?

Di fatto ci diventa palese l'insostenibilità della spiegazione meccanica del suono per tre ragioni. Anzitutto perché la spiegazione "meccanica" astrae dal fatto fondamentale che il suono appare 
esclusivamente in funzione di uno "strumento": l'organo uditivo. Ne consegue che il suono può solo venire spiegato se ci chiediamo di che cosa l'organo uditivo sensitivo sia "strumento" e quindi l'impossibilità di astrarre da questo fatto fondamentale. Dunque dobbiamo ammettere che il suono-in quanto si manifesta in funzione ad un "organo"- ha sempre già un significato in funzione di ciò di cui l'organo è strumento. In altri termini è palese che un suono ci appare solo entro l'ambito di un "codice" che si impone del quale il suono è indicazione cioé "voce," c solo conoscendo il codice in funzione al quale il suo significato appare e non astraendo da esso si puo intenderlo.

In terzo luogo: Il problema della determinazione razionale, causale, meccanica degli enti non è identica al problema del codice e ciò per la seguente ragione: il medesimo suono può-a secondo del codice-ottenere il significato di un richiamo, di una minaccia, di una proibizione. Il significato è indeducibile dal suono, è solo comprensibile nell'ambito del codice sensibile in funzione al quale appare il nostro mondo.

Questa constatazione ci fa comprendere l'importanza della cosiddetta tesi della "differenza ontologica" che nega l'identità del problema degli enti definiti razionalmente con quello dell'essere, inteso come un primo essente, e rifiuta la possibilità di partire dalla definizione razionale astratta degli enti per raggiungere il "codice" degli enti. Tale tesi è in netto contrasto con il pensicro tradizionale che si rifà alla interpretazione che Platone ha dato del filosofare socratico: astrazione razionale degli enti per raggiungere cosí il "codice" cioè l'Essere degli enti. È proprio la tesi della "differenza ontologica" che impone il compito di un nuovo filosofare di fronte a quello tradizionale.

Ma allora da dove prende le mosse il nostro filosofare se il partire dalla definizione astratta, razionale degli enti si rivela insufficiente? Come, dove si rivela il "codice" in funzione del quale appare il significato del sensibile reale se esso non è raggiungibile per mezzo dell'astrazione razionale? Ripeto: non in funzione di una spiegazione causale, meccanica, in quanto essa astrac dal fatto che il suono ri-suona esclusivamente come testimonianza dell'"opera," dell'"ergon," della quale è uno strumento.

Ma di che opera, di che "ergon" si tratta? Palesemente non di 
un'opera meccanica anche per la seguente terza ragione.

Il fondatore della fisiologia moderna, Johannes Müller, ha dimostrato che un medesimo stimolo-ad. es. una percussione-produce differenti apparizioni (phainomena-phainestai-apparire) specifiche per i vari sensi: per l'occhio la luce, per l'udito il suono, per l'organo gustativo il sapore.

J. Müller ha ulteriormente messo in luce che a loro volta differenti stimoli-percussione, un'azione elettrica, chimica-producono in ogni organo sempre l'apparire di un medesimo fenomeno, specifico per il singolo senso. Müller ha definito questa legge fisiologica come quella della "energia specifica dei sensi." (J. Müller, Über phantastische Geisteserscheinungen, Koblenz 1826, 4-5. Cfr. pure Handbuch der Physiologie des Menschen II, Koblenz 1849, 251. Cfr. pure Th. v. Uexkull, Die Physiologie des J. Müller und die moderne Medizin. Amtliche Wochenschrift der Medizin, 1958, Heft 28, 614).

Riprova della indeducibilità del significato dei fenomenti sensibili. L'indicazione di un suono, di un sapore, di un odore non può venire inteso in funzione di una considerazione meccanica perché questa è astratta. Ciò che appare per mezzo dei sensi e che costituisce l'ambito del nostro mondo si manifesta in funzione di strumenti, di organi di un "codice" che urge in essi entro i limiti del piacere e del dolore quale fonti delle passioni; come timore che ciò che preme in essi non venga adeguato; come speranza che tale opera venga compiuta. Si tratta dunque di corrispondere ad un appello abissale. È questo il senso delle parole di Novalis? "Tutto ciò che è visibile radica nell'invisibile, l'udibile nel non udibile, il sensibile nel non pensabile?" (Novalis, Das philosophische Werk, I, Studien zur bildenden Kunst, hrsg. von R. Samuel, Stuttgart 1981, 650, Frammenti, 710, trad. ital. di E. Pocar Rizzoli, Milano 1982). "Suoni" che non sono "voci" sono concepiti in funzione di una teoria meccanicistica che non può essere il presuposto di una teoria del linguaggio.

Dicevamo che non trattiamo qui il problema del "logo," del linguaggio, ma solo dei suoi elementi "vocali"—e quindi tanto meno della differenza che passa tra il linguaggio razionale, poetico e retorico-bensí solo la struttura della voce, del suono indicativo.

Ma già qui bisogna anzitutto porre la seguente domanda: il poeta si rifà forse alla passionalità delle voci, dei suoni indicativi? Non è forse egli a questo rigardo il "pro-feta," il "pre-annunciatore" 
nell'ambito del linguaggio di ciò che preme già nei sensi? Il poeta indica con la passionalità delle sue parole un mondo non razionale, misterioso, che si apre tra due oscurità: quella della "physis" abissale, non svelabile razionalmente e quella nella quale entra l'uomo quando fa l'esperienza di non ritrovare in funzione del codice sensibile il proprio ordine e quindi di dovere cercarne uno "nuovo," proprio dell'uomo?

Ricordiamo la seconda frase aristotelica nel Peri hermeneias: "Ciò che nella voce (psophos semanticos) si manifesta, sono simboli di ciò che l'anima patisce" (esti mèn tà én te phonè tov en te psyche pathematon symbola. Aristotele, Peri hermeneias 16 a).

Ciò che patiamo non sono gli enti ma ciò che in funzione dei sensi-entro i limiti di piacere e dolore-si impone sempre carico di significato. L'uomo vive esclusivamente sotto l'impeto di "segni indicativi," cioc̀ dell'Abissale di cui i sensi sono strumenti.

Ma possiamo veramente affermare che i suoni, le luci, gli odori ecc.-per mezzo degli organi sensibili di un razionalmente indeducibile e patito appello_-si manifestano sempre carichi di significato? Non veniamo continuamente disturbati scossi da rumori assordanti senza senso, abbagliati da luci accecanti?

Effettivamente siamo invece costretti ad ammettere che ogni apparizione sensibile-dalla piú tenue alla piú irruente-è carica di indicazioni passionali cioè di voci (phonai) anche e proprio quando per il suo apparire sembra priva di senso e di ogni passionalità.

Herder nel suo scritto Circa l'origine del linguaggio del 1770 scrive: "tutte le sensazioni, tra la forti e le piú forti, tra le forti, quelle dolorose, sensazioni del proprio corpo, come tutte le passioni della propria anima, si esprimono immediatamente nelle urla, nelle voci per mezzo dei suoni articolati. Tanto l'animale che soffre, quanto l'eroe Filottete, quando lo sopraffà il dolore comincerà a gemerc, a mugolare, abbandonato su un'isola sterile senza l'immagine, la traccia, la speranza di una caritatevole convivente" (Herder, Sämtliche Werke. Zur Philosophic und Geschichte, II. Teil, Tübingen 1806, 7). Tesi questa di Novalis che egli afferma nel primo paragrafo del suo scritto per poi abbandonarla in tutta la sua portata originaria nell'ulteriore sviluppo nella sua interpretazione dell'origine e struttura del linguaggio ricadendo nell'idealismo razionalistico.

Noia, paura, piacere e dolore, richiami e ripudi sono solo espres- 
sione, ammonimenti, segni indicativi, passionali, onde dei fenomeni sensitivi nelle quali, con godimento sensuale e disperazione, nuotiamo. $\grave{E}$ in questo ambito che si apre l'originario orizzonte nel quale esistiamo, che ci fa "sentire" i suoni "in-auditi," il visibile dell" invisibile," cioè la paradossia del nostro mondo.

Il suono senza senso è "inaudito" e proprio per ciò inquietante: la luce senza significato, senza una indicazione-è terrorizzante. Ogni essere organico ha cura, è pre-occupato per ciò che i suoi organi annunciano e ciò che per mezzo loro appare oscilla tra caos e cosmo, quest'ultimo come misura ed ornamento.

Per l'essere organico-cioè per quello che manifesta il proprio mondo attraverso i propri organi-non esiste un suono che non sia "voce," un sapore che non abbia un gusto differenziato. Solo il terrificante mito della preminenza della tecnica, della meccanica può affermare la tesi del carattere asettico di ciò che si manifesta con i sensi: cioè quel mondo astratto che il pensiero razionale ci propone.

Oltre il limite dei sensi domina il silenzio, il regno del muto e solo l'astrazione può parlare di suoni che non siano voci. In questo senso Leonardo da Vinci poteva affermare che il mondo è un unico essere vivente.

Herder afferma: "L'improvviso sopraffarci di una passione, l'imprevista gioia o dolore, segnano profondi solchi nell'anima: il sopraggiungere del sentimento della vendetta, della disperazione, della rabbia, dello spavento, dell'orrore, tutti si annunciano ed ognuno è specificamente differente. Quante specie di sensibilità sonnecchiano nella nostra natura, altrettanto sono le loro tonalità." Rinunciare a queste sensibilità significa morire. Le passioni premono come le onde tempestose, sciamano come gli storni: nel loro impeto tagliano e stracciano gli ondeggianti sipari del vento.

Herder sottolinea:

Le voci sono molto semplici e quando si articolano e vengono sillabate come interiezioni sulla carta, allora anche le piú contrapposte sensazioni ottengono una espressione. ... Lo scialbo Ah! è tanto voce di un amore doloroso quanto di una disperazione che si accascia, oppure: l'ardente Oh! è tanto espressione di una gioia improvvisa quanto dell'insorgente furore o della prorompente ammirazione. ... Le lagrime che appaiono nel torbido volto che anela a una consolazione, sono commoventi nell'immagine dello sconsolato: analizzate la lagrima isolata, sotto un microscopio, e non desidero nemmeno sapere cosa allora sia. Già il sospiro doloroso 
sembra liberare l'uomo, dando respiro all'intimorita sofferenza: sembra che una parte del dolore venga espirata per attingere dall'aria nuova forze riempiendo il sordo vento di un significato.

Dolore e gioia ri-suonano come un soffrire ed affermano una realtà originaria e misteriosa. "La corda vibrante realizza il proprio compito naturale, essa risuona, desta un'eco, anche se non c'è nessuno, anche se non spera né attende che qualcuno risponda." (Herder, op. cit., 8). Il carattere passionale del mondo sensibile è la radice del nostro mondo e tale passionalità ci rinvia costantemente alla sua indicativa radice abissale.

Herder vuole portarci sulle tracce dell'Abissale in funzione di cui gli strumenti palesano fenomeni: priorità dei sensi e quindi della sensualità.

Siamo cosí chini sul misterioso divenire dei fenomeni per ascoltare ed identificare il significato delle visioni, delle voci, delle immagini, nuotando sulle onde dei sensi giungiamo all'isola del nostro mondo sensibile, condannati come Filottete, e cerchiamo nella spuma dei marosi irrompenti sulla nostra spiaggia la molteplicità dell'apparire.

Con ciò-cosí si esprime Herder-si palesa la legge della natura: "Non sentire per te solo: che il tuo sentimento vibri . . . l'uomo che sembra isolato nelle tempeste nemiche dell'Universo non è isolato, non è cosí, egli è legato da una alleanza con la natura."

3.

Tre sono le conclusioni alle quali siamo giunti: il problema filosofico dal quale dobbiamo partire non è quello della definizione razionale degli enti ma il problema del loro Essere. L'Essere non si manifesta in funzione di un processo razionale ma originariamente nella passione di una codice "patito" e realizzantesi entro i limiti dei segni del piacere e del dolore. Precedenza quindi del problema della passione su quello della ragione. Capovolgimento quindi del filosofare tradizionale.

Di qui la necessità di porci la domanda: "dove," "come" patiamo la oggettività dell'Essere?

Dobbiamo, per rispondere alla domanda postaci, partire ancora una volta da un testo greco, il Cratilo, anche se questo non parte dal problema della "phonè," ma da quello dell'oggettività del linguaggio del "logo." Platone nel Cratilo pone la tesi: "Per ciascun 
ente (ekasto ton onton) vi è o un nome che gli compete per natura" (physei onomatos orthoteta. Platone, Kratilo 385 A4) oppure i nomi sono convenzionali (tine xunthemen xunthemenei, op. cit. A 6). Il nostro autore ripete la sua tesi: "La esattezza di un nome (orthotes onomatos) è o naturale (physei) o frutto di una convenzione" (ethei, Kratilo 384 D g).

Con la soluzione di questo problema avviene ciò che mai avremmo attesto dal pensiero di Platone: egli non parte piú dal problema degli onta - come in altri dialoghi-per giungere a mezzo di un processo razionale alla definizione dell'oggettività degli enti identificati con una idea astratta, razionale, ma prende posizione di fronte al relativismo sofistico partendo dell'esperienza, dal "pragma" cioè in funzione di ciò che si manifesta in funzione della praxis, dell'azione.

Platone pone in rilievo che la tesi sofistica circa il significato arbitrario, puramente convenzionale della parola, come viene sostenuta da Protagora, non può essere valida, perché dobbiamo distingere uomini "poneroi" e uomini "chreistoi," uomini che con il loro operare giungono solo a dolori, cioè incapaci, e uomini capaci (Kratilo 386 B 6).

Si tratta dunque di distinguere il nocivo dall'utilc. Ora dobbiamo riconoscere-incalza Platone-che ogni cosa ha una propria essenza, un proprio modo d'essere (delon de hoti auta auton ousian) e che quindi i "pragmata" non possono essere usati a nostro arbitrio (elkomena ana kai kato to emetero phantasmati, Kratilo 386 E 2): noi possiamo bruciare solo il combustibile, tagliare solo con ciò che è tagliente. Il "codice" sofferto in funzione al quale appare la realtà sensibile non è arbitrario, soggettivo, ma si impone ineluttabilmente.

Il pensiero greco distingue a questo riguardo radicalmente due forme di "agire": quella "poietica"—cioè propria della "poiesis"— e quella della "praxis": distinzione fondamentale che generalmente viene obliata credendo o confondendo che la "poiesis," il produrre, sia una "praxis," un agire.

Poiesis è sí un'azione, ma non originaria, è un mezzo, uno strumento per la realizzazione di un'opera, di un "ergon" che non gli è proprio, ragione per la quale l'azione si esaurisce, una volta realizzato l'opera, raggiunto il fine. La "poiesis" non è una attività in vista di se stessa, e quindi originaria, ma di altro, è mezzo di un fine che non le è proprio: perciò caduco quando questo è raggiunto. 
Praxis è invece una attività originaria la cui opera, "ergon" non sorge in vista di qualcosa d'altro, di un fine a lei alieno má che le è proprio, ella solo realizza la manifestazione di se stessa. "Le azioni che cercano il raggiungimento di un fine sono solo mezzi per raggiungere il fine ... perciò non possono venire considerate come 'praxis'... A Autentica prassi è solo quella che ha il fine, il limite in se stessa." (Aristotele, Metafisica 1048 b 18).

Ciò che ci rivclano i sensi-entro i limiti di piacere e dolore-e quindi i suoni, le luci, gli odori ecc.-non è un'opera, un "ergon" estraneo ai sensi—non è né un'"opera" meccanica nè un'opera "poietica," non un mezzo per raggiungere qualcosa ma "praxis" intesa come "parousia," palesarsi per eccellenza. Ciò che nei loro "erga" si manifesta è loro "en-ergia," il palesarsi dell'opera che è loro propria.

I suoni semantici schiudono il "teatro"-nel significato originario di questo termine-cioè il luogo del "vedere," del "theorein." L'incubo-proprio nel suo significato originario di ciò che incombe-è l'urgere del palesare, della "parousia." È proprio questo urgere che angoscia, che patisce l'essere organico nella sua funzione nutritiva e riproduttiva impostagli dalla "prassi."

$\mathrm{Ma}$ in cosa consiste il carattere metaforico dei segni sensibili? Esso diventa palese nella passione, nell'ambito della quale l'essente organico-tra i limiti di piacere e dolore-fa l'esperienza dell'oggettività di corrispondere o non corrispondere a ciò di cui i sensi sono "organi," strumenti. Tutto ciò che viene palesato è identico e non identico a se stesso in quanto rinvia a ciò di cui è una indicazione: metafora del mistero della realtà organica nelle sempre differenti "situazioni" e "voce" nella loro invisibilità.

Parlo di "situazioni" perché tutto ciò che i sensi manifestano ottiene, a secondo del "qui" e dell'"ora," cioc̀ a secondo del luogo e del tempo del premere dall'Appello abissale, un'altra indicazione. Platone nel Filebo afferma che ciò che in una situazione è piacevole in un'altra situazione appare spiacevole: "tote men aspasteos to de ouk" (Filebos 32 d 5).

Solo se la "poiesis" diventa un momento della "praxis" in tutta la sua passionalità si palesa la metaforicità del reale: in caso differente la "poiesis," il produrre diventa un gioco, una fuga dalla realtà. Tutto ciò che appare nel teatro originario-il cui sipario viene sollevato dai suoni, dalle luci, dagli odori, dai sapori-coincide con 
l'apparire della ineluttabilità della prassi che si palesa nella vita organica con i "suoni, segni indicativi," (psophoi semanticoi) la cui eco orginaria giunge fino agli elementi vocali. 